\title{
Subsequent Subarachnoid Hemorrhage from Clinically Unrelated Vertebral Artery Dissection after Thrombolytic Therapy
}

\author{
Min-jeong Bae, $\mathrm{MD}^{1}$, Sam Yeol Ha, $\mathrm{MD}, \mathrm{PhD}^{2}$ \\ ${ }^{1}$ Department of Neurology, Haeundae Paik Hospital, Inje University College of Medicine, Busan, Korea \\ ${ }^{2}$ Department of Neurology, Chung-Ang University Hospital, Chung-Ang University College of Medicine, Seoul, Korea
}

Thrombolysis administration poses certain safety issues in ischemic stroke patients with cerebrovascular changes that are vulnerable to hemorrhage. Furthermore, the lack of related studies has resulted in an unclear understanding of thrombolysis safety in ischemic stroke patients with intracranial dissection, including those involving the vertebral artery. This study describes a case of a 59-year-old female who developed subarachnoid hemorrhage from clinically unrelated vertebral artery dissection after thrombolysis. Histories of severe headache with posterior fossa involvement in patients receiving thrombolytic therapy may indicate careful assessment for intracranial vertebral artery dissection, even if the clinical picture of the patient suggests another arterial syndrome.

Key Words: Thrombolytic therapy; Vertebral artery dissection; Subarachnoid hemorrhage

\section{INTRODUCTION}

Thrombolysis administration poses certain safety issues in ischemic stroke patients with cerebrovascular changes that are vulnerable to hemorrhage. Although thrombolysis in cases of a non-ruptured aneurysm or extracranial artery dissection is relatively safe, ${ }^{1,2}$ its safety in ischemic stroke patients with intracranial artery dissection remains unclear due to a lack of evidence.

However, there have been a few cases of ischemic stroke caused by intracranial artery dissection that did not present with subsequent subarachnoid hemorrhage (SAH) after administration of recombinant tissue plasminogen acti- vator (r-tPA). ${ }^{3-5}$ Here, we report a contrasting case of subsequent SAH from unforeseen intracranial vertebral artery dissection (VAD) after thrombolysis in a patient with middle cerebral artery (MCA) syndrome.

\section{CASE REPORT}

A 59-year-old female presented to our institution 61 minutes after a clear onset of aphasia. She had experienced a severe headache on the previous day, followed by nausea and vomiting on the day of hospitalization. No history of recent trauma or any significant medical history was reported. Her blood pres-

\author{
Correspondence to: \\ Sam Yeol Ha, MD, PhD \\ Department of Neurology, Chung- \\ Ang University Hospital, Chung- \\ Ang University College of Medicine, \\ 102 Heukseok-ro, Dongjak-gu, Seoul \\ 06973, Korea \\ Tel: +82-2-6299-1485 \\ Fax: +82-2-6264-8272 \\ E-mail:samyeolha@gmail.com \\ Received: October 15, 2021 \\ Revised: December 30, 2021 \\ Accepted: January 3, 2022

\begin{abstract}
Copyright $\odot 2022$ Korean Society of Interventional Neuroradiology

This is an Open Access article distributed under the terms of the Creative Commons Attribution Non-Commercial License (http://creativecommons.org/licenses/by-nc/4.0) which permits unrestricted non-commercial use, distribution, and reproduction in any medium, provided the original work is properly cited.
\end{abstract}


sure on admission was $165 / 112 \mathrm{mmHg}$

Initial non-contrast computed tomography (CT) of the brain showed no evidence of intracerebral hemorrhage (Fig. 1A). She was diagnosed with an acute left MCA infarction after a significant mismatch was found between the perfusion CT and diffusion-weighted magnetic resonance imaging (DW-MRI) findings (Fig. 1B). The CT angiogram showed no occlusions in the large intracranial arteries, but it revealed a small non-ruptured aneurysm of the left MCA bifurcation accompanied by a slight dilation in the left intracranial vertebral artery (Fig. 1C). Her aphasia symptoms improved the time of arrival to our emergency room. However, her anomic aphasia corresponding to a National Institutes of Health Stroke Scale (NIHSS) score of 1 deteriorated to global aphasia
(NIHSS score, 5) at 5 hours 20 minutes after initial onset time. Consequently, we decided to administer late thrombolytic therapy considering fluctuating aphasia caused by misery perfusion in the inferior division of the left MCA, as well as due to the evidence of significant salvageable tissue on DW$\mathrm{MRI} /$ perfusion CT imaging and favorable outcomes in a recent trial on an extended time window for thrombolytic therapy. ${ }^{6}$ The last normal time to intravenous r-tPA time was 6 hours, which was 40 minutes post-drug administration for neurological deterioration. The patient's aphasia improved, showing an NIHSS score of 1 at 5 hours after r-tPA ( $0.6 \mathrm{mg} / \mathrm{kg}$ ) administration. Later, she complained of a severe headache having a numerical rating scale (1-10) score of 6 twice (6 hours and 9 hours 30 minutes) after administration of r-tPA,
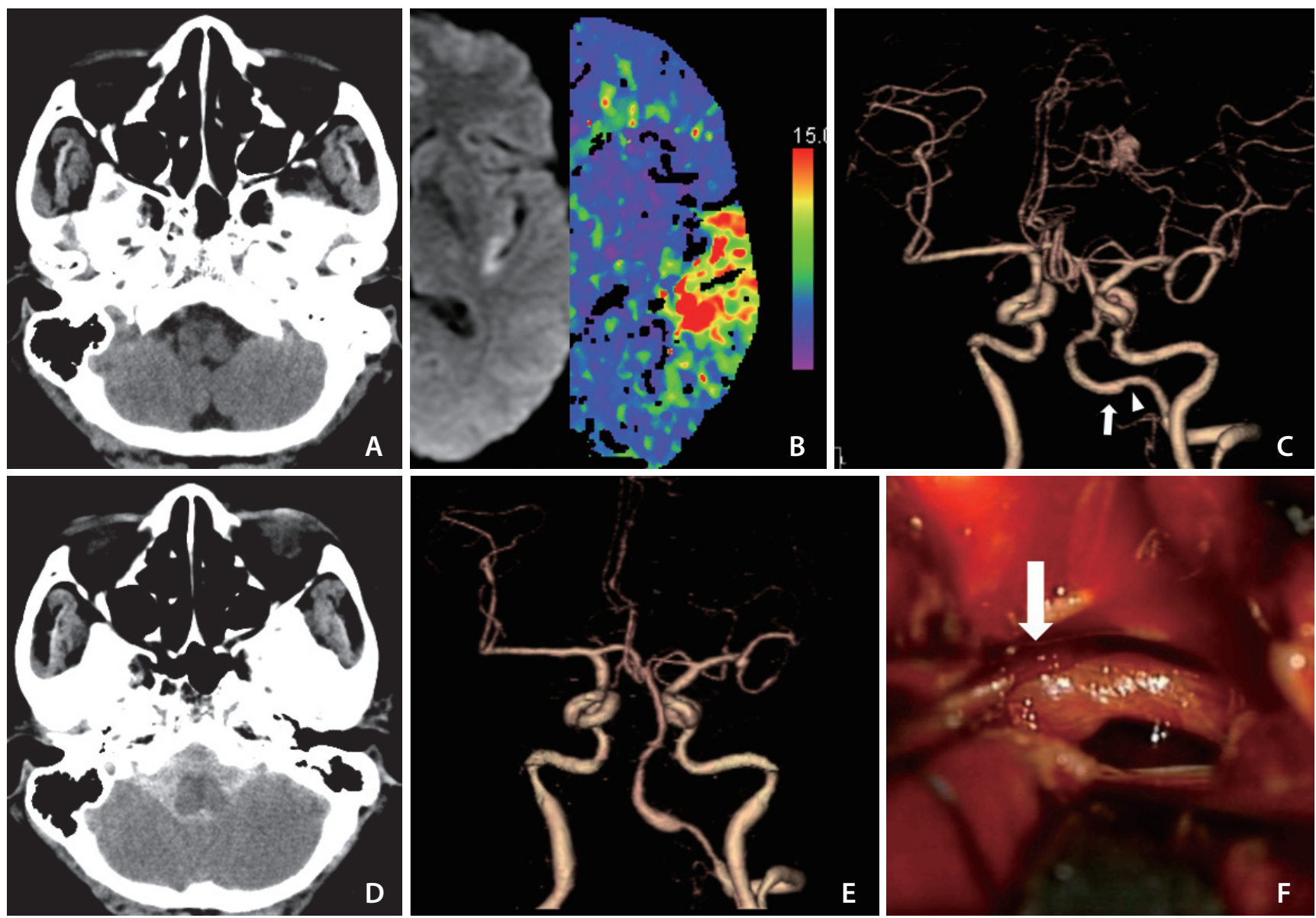

Fig. 1. A case of subarachnoid hemorrhage caused by the dissection of the left vertebral artery after thrombolytic therapy. (A) Initial brain computed tomography (CT) scan of a 59-year-old female patient shows no intracranial hemorrhage. (B) Diffusion-weighted magnetic resonance image and perfusion CT scan show significant mismatch on the left temporal lobe. (C) Initial CT angiography reveals mild dilation (arrow) and proximal narrowing (arrowhead) in the V4 segment of the left vertebral artery and a small non-ruptured aneurysm in the bifurcation of the left middle cerebral artery. (D) Repeated CT scans reveal a massive subarachnoid hemorrhage. (E) Repeated CT angiograms show more prominent dilation and narrowing in the V4 segment of the left vertebral artery than findings in $(\mathbf{C})$. (F) Intraoperative findings reveal a dissecting aneurysm with clots of this vertebral artery (arrow). 
with systolic blood pressure between 135 and $172 \mathrm{mmHg}$. Analgesics were administered, and she was kept under observation because her neurologic status was stationary. Subsequently, a seizure developed 10 hours 20 minutes after r-tPA administration, with blood pressure at 253/143 mmHg. After assessment of the seizure, brain CT and angiography were repeated. The imaging studies revealed SAH at the posterior fossa and intracranial VAD at an area clinically unrelated to the MCA syndrome (Fig. 1D, E), despite initial brain CT only showing subtle dilatation without hemorrhage around the intracranial vertebral artery. Intra-operative findings revealed a dissecting aneurysm with clots at the vertebral artery (Fig. 1F). Despite the surgical trapping of the dissecting aneurysm and decompression craniectomy for the occipital swelling and laminectomy on C1, the patient died of massive intracranial hemorrhage.

\section{DISCUSSION}

Although suitable treatment was provided for acute ischemic stroke, an unforeseen contingency developed during the hyperacute phase. In our case, SAH after thrombolysis was caused by a dissecting vertebral artery and not the aneurysm at the artery causing an infarction. In a meta-analysis of 5 case series studies on ischemic stroke patients with non-ruptured intracranial aneurysms treated with intravenous thrombolytic agents, the pooled rate of symptomatic intracranial hemorrhage was $6.7 \%$. ${ }^{1}$ Furthermore, the risk ratio of symptomatic intracranial hemorrhage after thrombolysis did not differ between acute ischemic stroke patients with or without a non-ruptured intracranial aneurysm. ' Similarly, a meta-analysis of 10 studies on patients with cervical artery dissection-related stroke found no statistical differences in symptomatic intracranial hemorrhage. ${ }^{2}$ Current clinical practice guidelines state that intravenous thrombolysis is reasonably safe and potentially recommended in patients with extracranial cervical dissection or non-ruptured aneurysms measuring $<10$ $\mathrm{mm}^{7}{ }^{7}$ However, thrombolysis safety in intracranial arterial dissection cases remains unknown and uncertain.?

Since vertebral and carotid arteries have different embryological origins, spontaneous dissections of these 2 arteries also differ. ${ }^{8}$ In an observational study analyzing 1,027 patients with new-onset spontaneous cervical artery dissection, SAH occurrence was 10 times higher in patients with spontaneous VAD than in patients with internal carotid artery dis- section (6.0\% vs. $0.6 \%)^{8}{ }^{8}$ Additionally, the intradural vertebral artery is more susceptible to rupture because it has a thinner tunica media and adventitia and contains fewer elastic fibers than other intradural arteries. ${ }^{9}$ Due to these histological differences, hemorrhagic intracranial arterial dissection occurs more often in the posterior fossa, which corresponds to the anatomic location of the vertebral arteries. ${ }^{8,10}$ Hence, the use of thrombolytic agents in patients with intracranial VAD may be associated with a higher risk of hemorrhagic complications than in those with other intracranial arterial dissections. Several studies have reported on the safety of intravenous thrombolysis in acute ischemic stroke due to cervical artery dissection ${ }^{2,5,11}$; however, these studies did not analyze intracranial VAD. Future studies should evaluate thrombolysis in relation to intracranial $V A D$, which might cause fatal bleeding, like our case. In our patient, the cause of the left MCA occlusion could not be determined due to incomplete study. The possible causes may be cardiogenic or involve rare simultaneous dissections of the MCA and vertebral artery.

A meta-analysis showed that headaches associated with ischemic stroke mainly had tension-type features with moderate to severe intensity in $6-44 \%$ of patients. ${ }^{12}$ Additionally, 2 single-center retrospective cohort studies found that headaches occurring during or immediately after intravenous thrombolysis via r-tPA administration did not reliably predict intracranial hemorrhage. 13,14 However, an acute, severe throbbing headache localized to the occipito-nuchal area is a typical symptom of VAD. This may serve as a warning sign, considering our experience with our patient who complained of a severe headache the day before admission and after r-tPA treatment. ${ }^{15}$ If we had attended to subtle dilation in the left intracranial vertebral artery on initial brain $\mathrm{CT}$ before r-tPA administration, we would have treated the patient using antiplatelet agents instead of thrombolytic agents, followed by the observation of the dissecting aneurysm for endovascular therapy and assessment for possible simultaneous dissections of the MCA and vertebral artery caused by a collagen vascular disease.

In conclusion, a detailed evaluation of a patient's vasculature through angiography may be indicated in ischemic stroke patients with a severe headache. Particularly, in cases with a clinical history of severe headache suggestive of posterior fossa involvement, careful assessment for intracranial VAD using angiography and additional imaging tests, such as susceptibility-weighted MRI would be beneficial. These imaging studies may be able to detect a small amount of 
hemorrhage or intramural hematoma in patients receiving thrombolytic therapy, even if the clinical picture of the patient indicates involvement of unrelated arteries.

\section{Fund}

None.

\section{Ethics Statement}

This case report was reviewed by the Institutional Review Board (IRB; Inje University Haeundae Paik Hospital Institutional Bioethics Committee) and conducted in accordance with the IRB policies. The IRB of Inje University Haeundae Paik Hospital waived the requirement for informed consent. Since consent for publication was not obtained due to the patient's demise, this case was given exemption by the IRB of Inje University Haeundae Paik Hospital.

\section{Conflicts of Interest}

The authors have no conflicts to disclose.

\section{Author Contributions}

Concept and design: SYH. Analysis and interpretation: MB and SYH. Data collection: MB. Writing the article: MB. Critical revision of the article: SYH. Final approval of the article: SYH. Overall responsibility: SYH.

\section{ORCID}

Min-jeong Bae: https://orcid.org/0000-0002-1125-161X

Sam Yeol Ha: https://orcid.org/0000-0002-3139-9160

\section{REFERENCES}

1. Goyal N, Tsivgoulis G, Zand R, Sharma VK, Barlinn K, Male S, et al. Systemic thrombolysis in acute ischemic stroke patients with unruptured intracranial aneurysms. Neurology 2015;85:1452-1458

2. Lin J, Sun Y, Zhao S, Xu J, Zhao C. Safety and efficacy of thrombolysis in cervical artery dissection-related ischemic stroke: a meta-analysis of observational studies. Cerebrovasc Dis 2016:42:272-279

3. Moon Y, Lee JH, Cho HJ, Roh HG, Choi JW, Chun Yl, et al. Intravenous thrombolysis in a patient with acute ischemic stroke attributable to intracranial dissection. Neurologist 2012;18:136-138

4. Yoshioka K, Mori T. Clinical and radiological difficulties to detect isolated MCA dissection before intravenous tPA therapy. J Stroke Cerebrovasc Dis 2019;28:104365
5. Bernardo F, Nannoni S, Strambo D, Bartolini B, Michel P, Sirimarco G. Intravenous thrombolysis in acute ischemic stroke due to intracranial artery dissection: a single-center case series and a review of literature. J Thromb Thrombolysis 2019;48:679-684

6. Ma H, Campbell BCV, Parsons MW, Churilov L, Levi CR, Hsu C, et al.; EXTEND Investigators. Thrombolysis guided by perfusion imaging up to 9 hours after onset of stroke. N Engl J Med 2019;380:1795-1803

7. Powers WJ, Rabinstein AA, Ackerson T, Adeoye OM, Bambakidis NC, Becker K, et al.; American Heart Association Stroke Council. 2018 Guidelines for the early management of patients with acute ischemic stroke: a guideline for healthcare professionals from the American Heart Association/American Stroke Association. Stroke 2018;49:e46-e110 Erratum in: Stroke 2018;49:e138, 2018;49:e233-e234

8. von Babo M, De Marchis GM, Sarikaya H, Stapf C, Buffon F, Fischer $U$, et al. Differences and similarities between spontaneous dissections of the internal carotid artery and the vertebral artery. Stroke 2013;44:1537-1542

9. Shin JH, Suh DC, Choi CG, Leei HK. Vertebral artery dissection: spectrum of imaging findings with emphasis on angiography and correlation with clinical presentation. Radiographics 2000;20:1687-1696

10. Park KW, Park JS, Hwang SC, Im SB, Shin WH, Kim BT. Vertebral artery dissection: natural history, clinical features and therapeutic considerations. J Korean Neurosurg Soc 2008;44:109-115

11. Zinkstok SM, Vergouwen MD, Engelter ST, Lyrer PA, Bonati LH, Arnold $\mathrm{M}$, et al. Safety and functional outcome of thrombolysis in dissection-related ischemic stroke: a meta-analysis of individual patient data. Stroke 2011;42:2515-2520

12. Harriott AM, Karakaya F, Ayata C. Headache after ischemic stroke: a systematic review and meta-analysis. Neurology 2020;94:e75-e86

13. Chaudhry SA, Kwon SY, Kneale H, Al Jajeh A, Hussain S, Razak A. Post-IV thrombolytic headache and hemorrhagic transformation risk in acute ischemic stroke. Neurol Clin Pract 2016;6:22-28

14. James B, Chang AD, McTaggart RA, Hemendinger M, Mac Grory B, Cutting SM, et al. Predictors of symptomatic intracranial haemorrhage in patients with an ischaemic stroke with neurological deterioration after intravenous thrombolysis. J Neurol Neurosurg Psychiatry 2018;89:866-869

15. Kim JG, Choi JY, Kim SU, Jung JM, Kwon DY, Park MH, et al. Headache characteristics of uncomplicated intracranial vertebral artery dissection and validation of ICHD-3 beta diagnostic criteria for headache attributed to intracranial artery dissection. Cephalalgia 2015;35:516-526 